\title{
CERÁMICA: MATERIALIDAD Y SIMBOLISMO. EN TORNO A LOS SIGNIFICADOS QUE ENCIERRA EL CONSUMO DE CERÁMICA IMPORTADA EN ASTURIAS \\ POTTERY: MATERIALITY AND SYMBOLISM. ABOUT THE MEANINGS OF THE IMPORTED POTTERY CONSUMPTION IN ASTURIAS
}

Miguel Busto Zapico* Universidad de Granada

\section{Resumen}

A través del estudio de 3.220 piezas cerámicas con una cronología que se extiende desde el siglo XIII al XVIII y que han sido recuperadas en diferentes excavaciones arqueológicas realizadas en el Principado de Asturias hemos sido capaces de obtener datos de índole social. De este modo, ofreceremos una síntesis de la evolución de consumo de la cerámica en Asturias desde la Baja Edad Media y durante la Edad Moderna. Nos fijaremos en alguno de los significados y las pautas sociales que nos señala su consumo. Por último, estudiaremos determinadas piezas cerámicas que fueron utilizadas por las élites sociales como un instrumento en el que reflejar su estatus dominante. Moderna.

Palabras clave: Cerámica, comercio, sociedad, Arqueología, Edad

\section{Abstract}

Through the study of 3,220 pottery pieces ranging in date from $13^{\text {th }}$ century to $18^{\text {th }}$ century that have been found in different archaeological excavations carried out in Principality de Asturias, we have been unable to find social data. In this way, we provide a summary of the evolution of the pottery use and consumption in Asturias from the Late Middle Ages to the Early Modern Period. We will focus on some of the meanings and social patterns point by the uses. Finally, we will study some of the pottery pieces that were used by the social elites as an instrument to reflect their ruling status.

Keywords: pottery, trade, society, Archaeology, Early Modern Period. 


\section{Introducción ${ }^{1}$}

En este trabajo analizamos la significación que encierran tras de sí algunas piezas cerámicas recuperadas en excavaciones arqueológicas realizadas en el Principado de Asturias. Hemos analizado una muestra altamente representativa formada por 3.220 cerámicas procedentes de unos contextos arqueológicos con buena fiabilidad estratigráfica y con unas cronologías que abarcaban desde el siglo XIII hasta el XVIII ${ }^{2}$. La ciudad de Oviedo es el núcleo que mayor número de piezas nos ofrece $(59,44 \%)$, seguida de Gijón (18,35\%), Cangas del Narcea (7,98\%), Avilés (6,09\%), un pecio hundido en la Ría del Eo (5,43\%), Castropol (1,99\%), Grado (0,44\%) y, por último, Villaviciosa $(0,28 \%)$.

De Oviedo proceden la gran parte de los yacimientos seleccionados, en concreto 30; seguido de Avilés con ocho y Gijón con siete (Fig. 1). El resto de los núcleos aportan un yacimiento cada uno. En esta selección nos hemos preocupado de elegir ámbitos que fuesen diferentes entre sí para conocer las posibles discrepancias en el consumo cerámico habidas entre ellos, aunque será Oviedo la ciudad que centre nuestro interés. De este modo, tenemos materiales procedentes de núcleos urbanos como son Oviedo, Gijón o Avilés; de entornos de menor entidad poblacional, como pueden ser Castropol, Villaviciosa o Grado; de un lugar monástico del interior de Asturias, tal es el caso del monasterio de San Juan Bautista de Corias, en Cangas del Narcea.

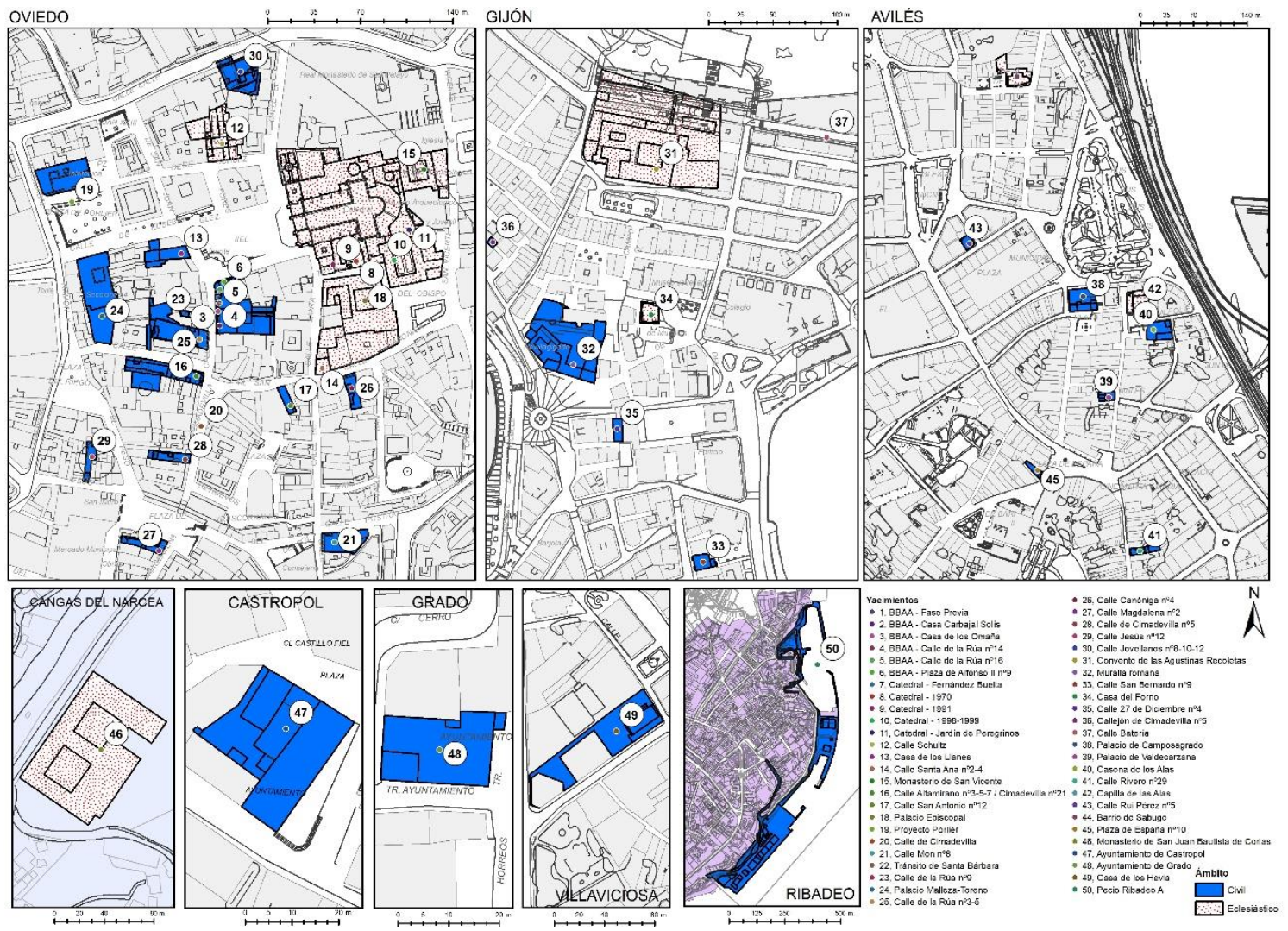

Fig. 1. Localización de las 50 excavaciones arqueológicas analizadas. 
A ellos habría que añadir un pecio hundido en la Ría del Eo. Al mismo tiempo, se buscaba tener una representación amplia del sustrato social asturiano con ámbitos palaciegos, grandes casas urbanas o habitaciones relacionadas con el cabildo y también hábitats más modestos, como casas en zonas periféricas. Todo ello con el objetivo de conocer el consumo cerámico, sus diferencias y su evolución.

\section{Síntesis de la evolución del consumo de cerámica importada en Asturias desde el siglo XIII al XVIII.}

El consumo de cerámica importada en Asturias desde el siglo XIII hasta el XVIII está marcado por una serie de alfares que irán cambiando y sucediéndose a lo largo de este amplio periodo ${ }^{3}$ (Fig. 2. A). Serán principalmente los centros productores franceses, sevillanos, italianos, holandeses, talaveranos y castellanoleoneses, los que destaquen en Asturias.
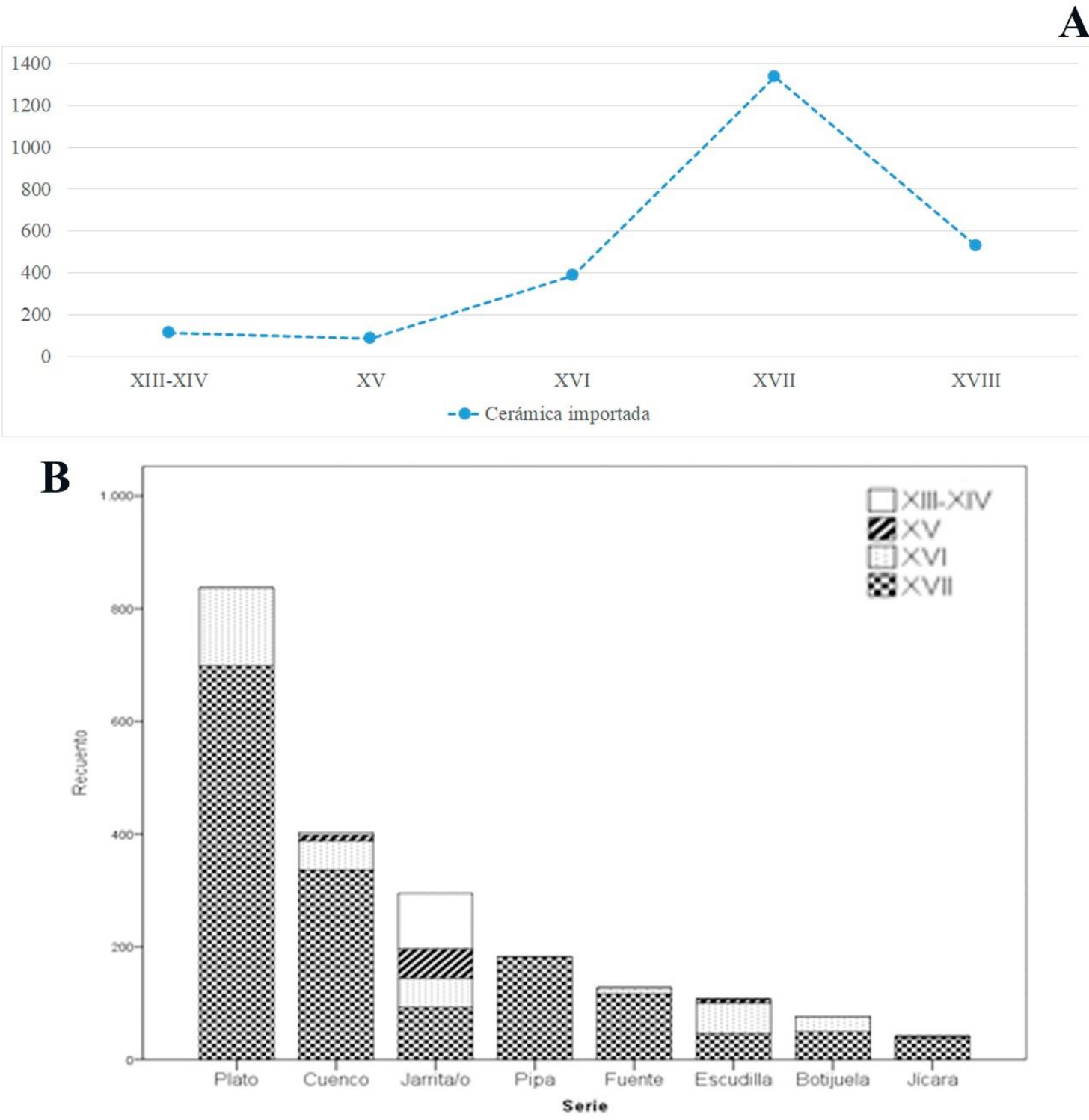

Fig. 2. A: Evolución del consumo de cerámica importada en Asturias entre los siglos XIII-XIV al XVIII. Fig. 2. B.: Series cerámicas más consumidas en Asturias entre el siglo XIII y el XVII. 
Durante la Baja Edad Media las cerámicas francesas son las que tienen la hegemonía en el comercio asturiano. En el siglo XV comienza su caída al mismo tiempo que suben las importaciones procedentes de Sevilla. Tendremos que esperar al siglo XVI para observar el gran auge de las producciones sevillanas. En el quinientos, las cerámicas sevillanas inundan los mercados asturianos y ningún otro centro puede hacerles sombra. Tan solo los centros italianos podrían competir entre las clases más adineradas, con unas producciones de lujo al alcance de unos pocos.

En el siglo XVII la situación cambia y la hegemonía de Sevilla es sustituida por las producciones provenientes de los Países Bajos y Talavera de la Reina, quedando el centro andaluz en una posición secundaria. El seiscientos es una época de florecimiento en Asturias en lo que se refiere a la gran diversidad observable en las cerámicas que llegan a sus puertos. Ningún otro periodo ofreció a los consumidores asturianos una variedad tan grande. Junto a las producciones ya citadas de piezas holandesas y talaveranas, tenemos que añadir cerámicas portuguesas, inglesas y alemanas, junto con las provenientes del País Vasco. Este esplendor se extendería solo una centuria para pasar a una situación muy diferente en el siglo XVIII.

En el setecientos las grandes ventas de cerámica europea cesan de forma casi total y el mercado cerámico se centra en las producciones peninsulares ibéricas. Sevilla vuelve a ser un centro importante y a éste se le suman producciones llegadas de la meseta. Observamos cómo la fabricación de cerámica esmaltada entra en crisis ante la competencia que ejercen las nuevas producciones industriales.

\section{La cerámica y los significados que encierra su consumo.}

La significación es la idea, imagen o concepto que evoca cualquier signo o fenómeno interpretable. En nuestro caso, creemos que la significación de las cerámicas trata acerca del valor que, entre la Baja Edad Media y durante la Edad Moderna, los asturianos les dan a determinadas piezas de su vajilla. Muchas de estas piezas son usadas para colgar de las paredes a modo de decoración. Otras están repletas de huellas de uso y muy desgastadas, lo que nos indica una utilización muy intensa y un significado distinto. En este trabajo nos centraremos, principalmente, en la vajilla esmaltada también conocida como cerámica estannífera o loza, es decir, aquella que tiene un acabado blanco conseguido con el uso de estaño. La cerámica esmaltada en la Edad Moderna era un nuevo objeto de consumo y, al mismo tiempo, un bien no estrictamente necesario que podía ser fácilmente vendido en una sociedad con mayores posibilidades económicas ${ }^{4}$.

Para que un producto fuese demandado era necesario que se verificase un cambio en las costumbres. En el caso de la cerámica, estos cambios no solo afectarán a las élites civiles o religiosas, sino también a una clase urbana que iba creciendo desde la Edad Media. Bien es cierto que las élites serán las que potencien los cambios y las que irán a la vanguardia en cuanto a consumo de cerámica importada se refiere. El resto de los grupos sociales tratarán de adaptarse a esas nuevas modas y formar parte de ellas. 
El consumo de cerámica importada en Asturias fue causado por varios factores. Uno de ellos, tal y como se ha demostrado en otras zonas de la Península Ibérica, tuvo que ver con el auge en el uso del servicio individual de mesa ${ }^{5}$. En la Edad Media las piezas que se utilizaban en la mesa tenían una función práctica, las formas eran limitadas ${ }^{6}$ y el material utilizado más a menudo era la madera, siendo escasos los objetos de cerámica y muy escasos los de metal. Solo alguna parte de la nobleza podía permitirse la vajilla de plata y de oro, que representaba un símbolo de opulencia. Esta tendencia es la que observamos en el consumo de cerámica importada en los siglos XIII y XIV en Asturias, donde las piezas cerámicas importadas constituían un escaso porcentaje ${ }^{7}$. Eran principalmente formas dedicadas a contener vino rematadas con una cubierta vítrea de color verde que las hacía muy sugestivas, tratando de acercarse a las formas metálicas.

Durante la Edad Moderna se produjeron cambios en los hábitos alimenticios, lo que desembocaría en la necesidad de crear un menaje distinto. Hay mayor variedad de comida preparada debido al auge de nuevos productos agrícolas y el acceso a una mayor cantidad de carne y verduras ${ }^{8}$. Cada comensal va a tener su propio plato, que se cambiaba conforme se sucedían los manjares. Junto al servicio individual, en las mesas de las clases nobles, comenzaron a utilizarse los cubiertos, tenedores, cuchillos y servilletas, tal y como ponen de manifiesto algunos inventarios de mercaderes ${ }^{9}$. La preparación de la comida, el auge de la etiqueta y las buenas maneras se volvieron un componente esencial del estatus del individuo en una sociedad siempre más jerárquica ${ }^{10}$.

Los años finales del siglo XV y todo el quinientos fueron testigos de importantes cambios. Hay un progresivo refinamiento de las costumbres y es en estos momentos cuando se difunden los manuales de urbanidad y buenos modales ${ }^{11}$. Los cambios que se aprecian comienzan a ser palpables a partir del siglo XVI, motivados por el ascenso al trono de la dinastía de los Austria ${ }^{12}$. Muchos de los ministros y asesores que llegaron con los primeros monarcas eran flamencos e introdujeron en la corte castellana nuevos gustos en el ornato decorativo, en las vestimentas y también en los usos culinarios, que pronto fueron imitados por toda la sociedad13. Estos cambios hicieron necesaria la normalización de las formas cerámicas que ya existían y la creación de otras nuevas. Como consecuencia aumentaron las piezas que se utilizaban y se ponían en la mesa, cada una con una función más específica ${ }^{14}$. Como ejemplo de la alimentación y, como consecuencia, de las necesidades de menaje cerámico que podrían tener personajes de alta nobleza en el siglo XVII, nos puede servir lo resuelto por el rey Felipe IV el 13 de junio de 1631 concerniente a las percepciones que recibiría el ayuda de cámara: "resuelvo ahora que de aquí adelante los días semejantes se dé al que lo fuere (ayuda de cámara) tres tortillas, media açumbre de bino, tres libras de fruta de prinçipio y postre conforme al tiempo; y si fueren pasas y almendras la mitad que hasta ahora se les a dado, veinte y quatro huevos, libra y media de peçes, libra y media de truchas si las huviere u otro pescado fresco o escabeche en su lugar, un quarterón de açúcar con el açeite necesario para aderezar esta vianda, y el día que fuere de ayuno se le dará doze guevos y una libra de pescado fresco menos sin otra cossa... y que los días que yo comiere de pescado 
no se dé plato a nayde si no fuere del remanente de mi mesa como se haze el día de carne"15. Los alimentos que en esta resolución se enumeran hacen necesario un buen número de piezas cerámicas. Jarras, jarritas, cuencos o escudillas para servir y consumir el vino, las pasas, las almendras o el azúcar. Platos para las tortillas, el postre, los huevos, el pescado o la carne. Fuentes para las frutas; y, por último, aceiteras o jarras para el aceite. Estas tendencias son observables en la muestra cerámica analizada. Así, en Asturias, las series cerámicas más producidas y demandadas son los platos y los cuencos, piezas de consumo individual, seguidas por las jarritas o jarritos (Fig. 2. B). Para encontrar una pieza de consumo grupal tenemos que dirigirnos al quinto lugar. Se trata de las fuentes, que serían unas piezas en donde se mostrarían los alimentos en la mesa para que cada comensal se sirviera de manera individual (Fig. 2. B).

En Asturias el servicio de mesa se institucionalizó y se amplió (Fig. 3. A), debido a los nuevos productos exóticos procedentes de América, Lejano y Medio Oriente, como el chocolate, el té o el café16. Prueba de que estos cambios se dan en Asturias y que se consumen este tipo de alimentos es la aparición de jícaras ${ }^{17}$, porcelanas $\operatorname{chinas}^{18}$ y piezas mexicanas ${ }^{19}$, en los registros arqueológicos del siglo XVII. La aparición en los mercados de productos exóticos procedentes de lejanos lugares fue básica a la hora de observar cómo los usos en la sociedad se van modificando ${ }^{20}$. Las clases sociales acomodadas asturianas, principalmente entre los siglos XVI y XVII incorporaron en sus ajuares cerámicos toda una serie de producciones de gran riqueza y de lugares dispares $^{21}$ que no podemos entender sin la inserción de Asturias en los mercados. El aumento de la demanda de cerámica importada dependió también de la mejoría en la calidad de los productos. Con la fabricación de productos de mejor calidad, aumentó su aceptación. Una de las mejoras de estos productos con respecto al menaje utilizado en momentos anteriores pudo ser, según se atestigua por los datos documentales italianos, que las piezas revestidas no daban sabor a la comida ${ }^{22}$, lo que las hizo más apreciadas. Este hecho no pudo ser ajeno a los consumidores asturianos, dado que las cerámicas que importan son mayoritariamente destinadas al consumo y servicio de alimentos (Fig. 3. A). Por tanto, reservadas al uso en la mesa y casi en su totalidad revestidas, tanto vidriadas (uso de plomo) como esmaltadas (uso de estaño). En las piezas destinadas al consumo de líquidos, como las jarritas o los jarritos, observamos una mayor heterogeneidad en cuanto a sus revestimientos (Fig. 3. B). Por el contrario, en los platos, cuencos y escudillas domina de manera aplastante la cubierta estannífera (Fig. 3. B). Sin embargo, no debemos olvidar que al mismo tiempo éste es un momento en que las poblaciones europeas alaban no sólo el color, sino el sabor y el olor de estos objetos de barro 23 . Por eso se consumirán en Asturias algunos búcaros o cerámica vermelha portuguesa ${ }^{24}$. 


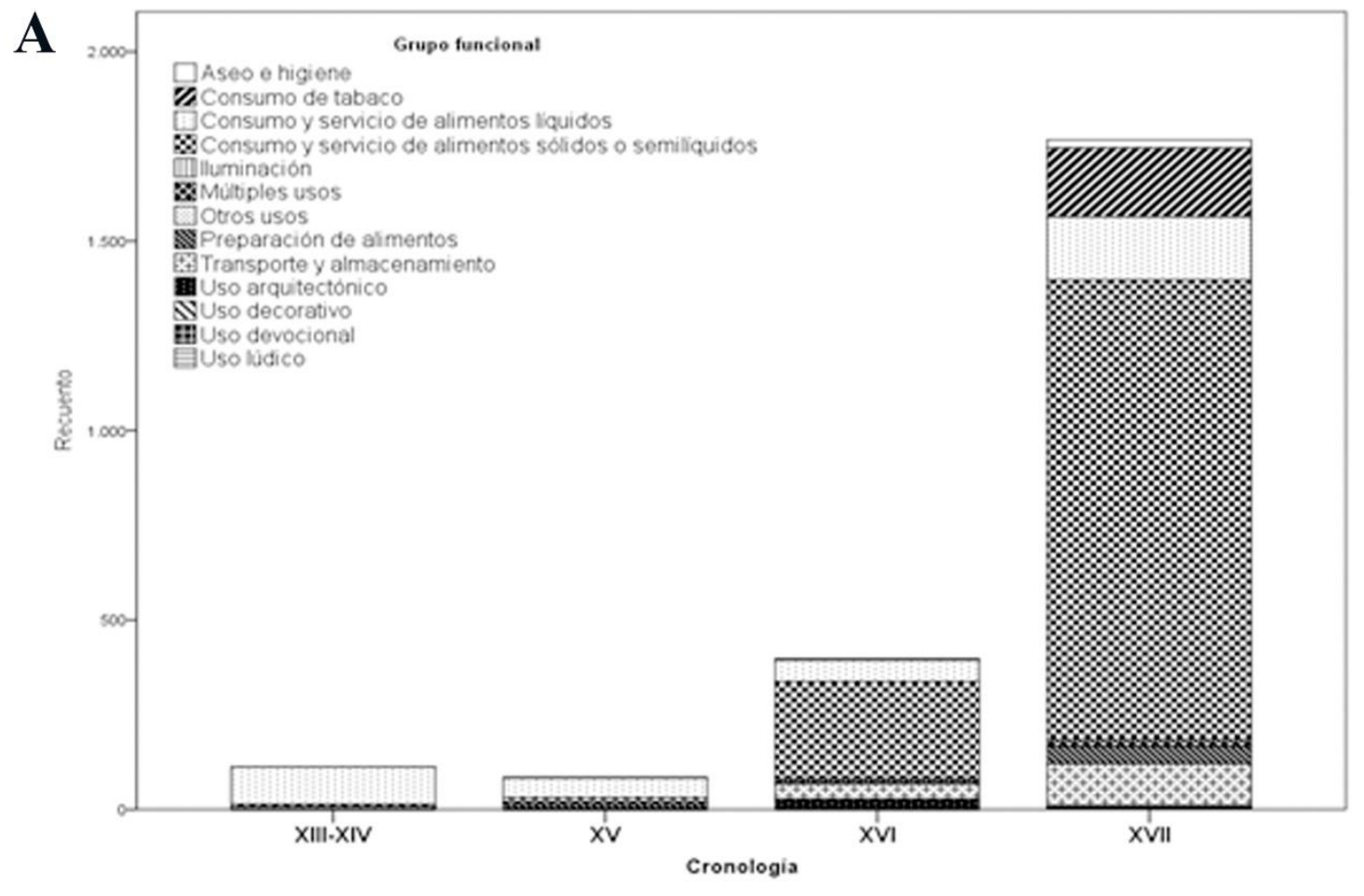

B

\section{Grupo funcional}

Consumo y servicio de alimentos liquidos

Consumo y servicio de alimentos sólidos o semiliquidos
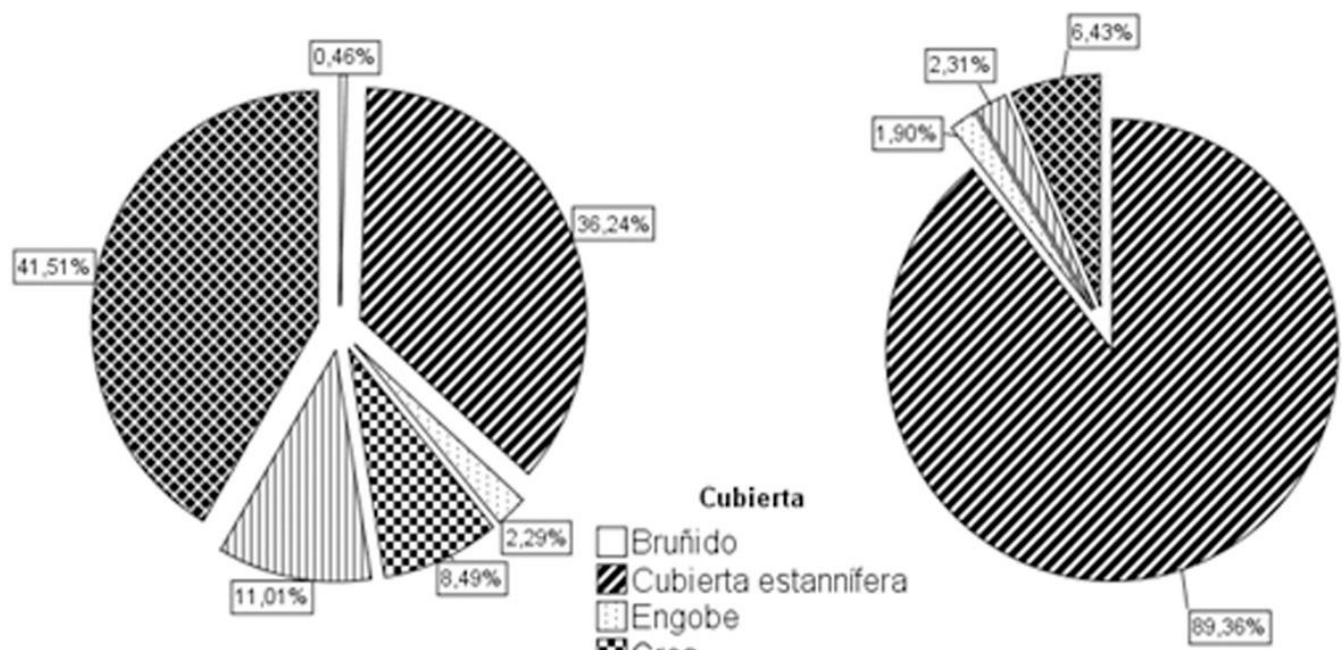

QGres

IIIIS Sin cubierta

Vidriado

Fig. 3. A: Grupos funcionales de cerámica importada en Asturias desde el siglo XIII al XVII. Fig. 3. B: Tipos de cubierta dentro de la cerámica de mesa consumida en Asturias. 
Estas piezas fueron muy apreciadas por el color, el brillo, las formas, la decoración, el olor y el sabor que otorgaban a los líquidos ${ }^{25}$. El sabor y aroma que daban al agua que probablemente contuvieron, hizo que estas piezas fuesen conocidas como "loza de olor" 26 . En ocasiones estas piezas podían ser ingeridas, sobre todo en el mundo femenino ${ }^{27}$. Las damas las comían, después de beberse el agua, ya que consideraban que su ingesta daba a la piel un tono pálido muy apreciado en la época ${ }^{28}$. Esta costumbre recibía el nombre de “comer barros" y las piezas se llegarían a fabricar en un tamaño reducido para poderlos incorporar, como un adorno más, en las vestimentas, y así tenerlas a mano en todo momento 29 .

En la segunda mitad del siglo XVII, en Oviedo nos encontramos a las oligarquías urbanas, que serán las grandes consumidoras de productos de importación entre los que se encontrarían las cerámicas. De este modo, en el padrón de vecindad de Oviedo de 1664, tenemos a un caballero de la Orden de Santiago, Gaspar de Caso, hidalgo de la familia y linaje de $\mathrm{Caso}^{30}$. En la calle Cimadevilla tiene su casa Antonio de Heredia y Alonso, y su hijo, ambos caballeros del hábito de Santiago ${ }^{31}$. También en Cimadevilla tenemos a Gonzalo de Solís que es alférez mayor de Oviedo ${ }^{32}$. Pedro Gamarra, oidor en la Real Chancillería de Valladolid, gobernador y capitán de Oviedo, tiene su casa en Cimadevilla en $1664^{33}$. En 1670 , en la calle de la Platería, tenemos a otro caballero de la Orden de Santiago, se trata de Ramiro de Buergo Baldez ${ }^{34}$. En la calle de la Rúa, desde 1670, vive Sebastián Vigil de la Rúa, caballero de la Orden de Calatrava, regidor de Oviedo y marqués de Santa Cruz de Marcenado ${ }^{35}$. En 1692, tiene su casa en la Rúa, Rodrigo de Cienfuegos Estrada, conde Marcel de Peñalba ${ }^{36}$.

Igualmente, descubrimos en el centro de Oviedo en el mismo periodo, profesiones liberales, que bien podrían estar relacionadas con la importación de cerámica. En los padrones de vecindad de Oviedo de 1664 y 1670, nos encontramos con dos boticarios en la calle de Cimadevilla, de los que podemos esperar que utilizasen albarelos de cerámica para almacenar y conservar sus drogas, como los descubiertos en las excavaciones arqueológicas ${ }^{37}$. Se trata de Cosme Paxente y Agustín Alonso de la Torre ${ }^{38}$. Es importante el buen número de mercaderes afincados en el centro de Oviedo en la segunda mitad del seiscientos. Hemos rastreado varios nombres como Bartholomé Piquero Argüelles ${ }^{39}$, Bernardo Piquero ${ }^{40}$, Francisco Tejero ${ }^{41}$, Lucas Menéndes ${ }^{42}$, Antonio Gómez ${ }^{43}$, Francisco Fernández Álvarez ${ }^{44}$, Francisco de la Vega ${ }^{45} \mathrm{o}$ Josef de Toro ${ }^{46}$. Este último aparece ligado a la ciudad de Toro, de la cual hemos atestiguado cerámicas ${ }^{47}$. Creemos que es probable que, entre las mercancías vendidas y compradas por estos mercaderes, en alguna ocasión, se encontrasen piezas cerámicas.

También en la segunda mitad del siglo XVII podemos rastrear familias que no son de origen asturiano o que residen fuera de Asturias. Tal es el caso de Vicente Fernández, residente en la villa de Madrid, que tiene una vivienda en la calle de la Rúa en $1670^{48}$. Un caso similar es el de Gregorio Menéndez ${ }^{49}$, citado en el padrón de 1692 como residente en Castilla. Estos datos nos señalan los movimientos de personas dentro del territorio peninsular y también procedentes de otros reinos. Estos flujos traerán consigo nuevas 
ideas, nuevos gustos y también nuevos productos que pueden ayudarnos a enmarcar la llegada de cerámicas importadas. La calle de la Rúa debió de comportarse como una zona cosmopolita de Oviedo. Así, tenemos en el padrón de vecindad de 1692 a Ricardo Galbez, de nacionalidad irlandesa ${ }^{50}$. Del mismo modo, este hecho puede señalarnos cómo algunos movimientos de mercancías y en nuestro caso, de piezas cerámicas, podrían estar relacionados con los movimientos de personajes concretos.

\section{Cerámicas: entre la materialidad y el simbolismo.}

En las páginas siguientes trataremos de dilucidar la relación que existió entre algunas cerámicas y sus consumidores. Determinadas producciones halladas en Asturias con cronología de la Edad Moderna actúan de manera clara y buscada como el reflejo de una clase social específica. A continuación, nos fijaremos en una serie de casos concretos que conectan la materialidad arqueológica, es decir, la pieza cerámica, con algún personaje, alguna familia o algún grupo social concreto. Estas piezas van más allá de una mera pieza de vajilla para convertirse en un símbolo.

\section{a. La vajilla del obispo}

Entre los materiales recuperados en las excavaciones realizadas en el entorno de la Catedral de San Salvador de Oviedo ${ }^{51}$ destacan cuatro cuencos que habían sido pintados con dos escudos heráldicos y poseían una inscripción que recorría el borde externo (Fig. 4. A). Han sido analizados y clasificados como Talaverana Heráldica - Cuenco IA y Talaverana Heráldica - Cuenco IB, distinguiendo de este modo los dos motivos heráldicos que se desarrollan ${ }^{52}$. Estamos ante unas piezas que, sin ninguna duda, pertenecieron a un obispo concreto, con poder y relevancia.

Con los datos emanados de los dos motivos heráldicos, de la inscripción en el borde, de las características técnicas y del contexto de aparición de estas piezas, tratamos de rastrear su posible propietario. Lamentablemente, el contexto arqueológico de la tipología Talaverana Heráldica - Cuenco I no era del todo clarificador. Estas piezas proceden de la intervención de Fernández Buelta en la Catedral de Oviedo y no tienen un contexto arqueológico estratigráfico bien definido ${ }^{53}$. Por ello debimos de centrar nuestro análisis en las propias piezas. A través del estudio ceramológico observamos que, desde un punto de vista técnico y decorativo, la Talaverana Heráldica - Cuenco I estaba en estrecha relación con las producciones decoradas en azul de la serie heráldica Austria, que se elabora en Talavera de la Reina en el siglo XVII ${ }^{54}$.

La inscripción desarrollada en el exterior del borde en Talaverana Heráldica - Cuenco I, una vez reconstruida a través de todos los fragmentos, es la siguiente: FR, ILDEPHONSUS EPISCOPUS OVETENSSIS, DHS (Fig. 4. B). De ella podemos extraer al menos tres datos claros: se refiere a un fraile, obispo de Oviedo y de nombre Ildefonso. Si a esto le unimos los datos del análisis ceramológico, conseguimos circunscribir nuestra búsqueda al siglo 
XVII. Al examinar el listado de obispos de Oviedo en dicha centuria, ninguno cumple totalmente estas características. Aun así, nos fijamos en fray Alonso de Medina y Salizanes que fue obispo de Oviedo entre 1669 y $1675^{55}$. Si bien se llama Alonso, podríamos estar ante una latinización del nombre. De todos los obispos del siglo XVII era Alonso de Medina y Salizanes el que se acercaba más al personaje buscado.

A
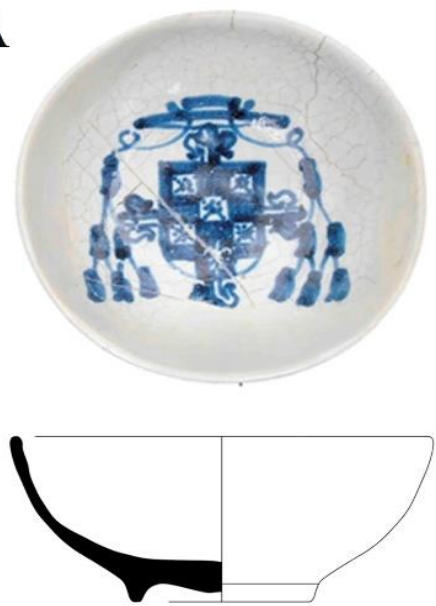

B
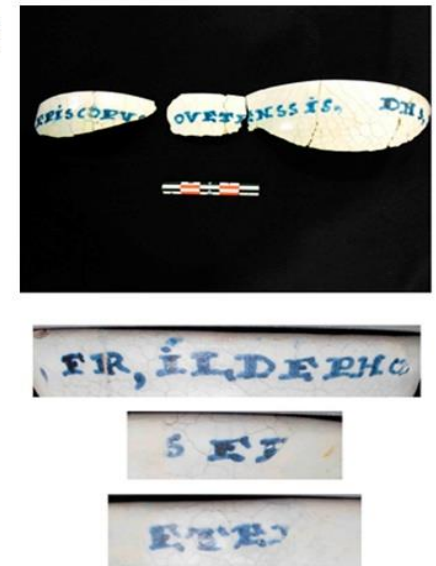
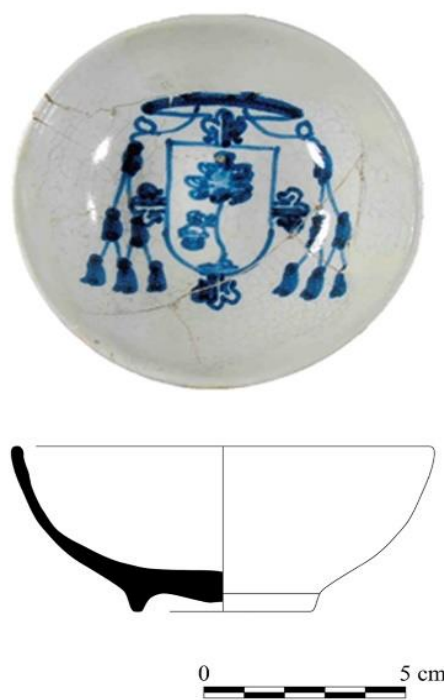

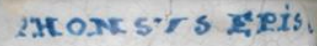

$\cos$

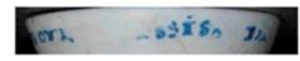

corers ord.

$-35 i s=$ toa

a. GDE2

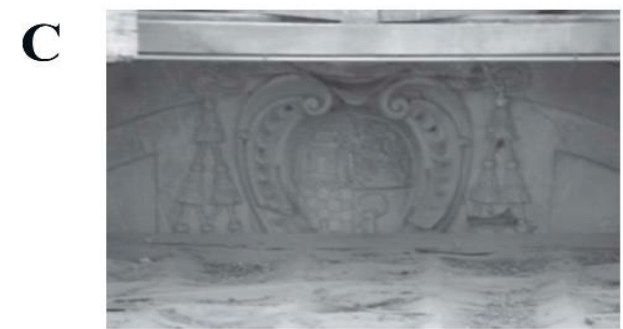

Fig. 4. A: Dibujo, acabado y decoración de Talaverana Heráldica - Cuenco IA (derecha) y Talaverana Heráldica - Cuenco IB (izquierda). Fig. 4. B: Fragmentos con inscripción en Talaverana Heráldica - Cuenco I. Fig. 4. C: Escudo del obispo don Alonso de Medina y Salizanes; nave axial de la mezquita fundacional de Abd al-Rahman I (JORDANO BARBUDO, María Ángeles, "La intervención de...", cit., p. 17, Fig. 3). 
Es, en este momento, cuando los motivos heráldicos cobran importancia en nuestras indagaciones porque a través de ellos podemos comprobar si tras FR, ILDEPHONSUS EPISCOPUS OVETENSSIS se esconde el obispo Alonso de Medina y Salizanes. Tras ser obispo de Oviedo, Salizanes es nombrado obispo de Córdoba, cargo que ostentará entre 1675 y $1685^{56}$. Se convierte en un obispo relevante en la historia eclesiástica cordobesa, que va a llevar a cabo labores de reforma en la Mezquita-Catedral57. En estas intervenciones el obispo Salizanes colocará sus escudos en lugares destacados, llegando hasta nuestros días.

El escudo heráldico de Salizanes representa el linaje de sus padres, don Gaspar de Medina Requejo y doña Josefa Sánchez Salizanes ${ }^{58}$. Se trata de un escudo con el campo cuartelado (Fig. 4. C); en el primer cuartel en campo de plata una torre de oro sobre ondas y sobre la torre una bandera de gules. Bordura de azur y plata; en el segundo cuartel en campo de oro, cinco leones en sotuer de plata, linguados de gules. En el tercer cuartel ajedrezado de plata y gules, y sobre los de plata aspas o husos, y en uno de ellos una flor de lis. Por último, en el cuarto cuartel en campo de azur un árbol de sinople con una mano saliente y una caldera ${ }^{59}$. Los motivos desarrollados en el cuartel tercero y cuarto coinciden con la heráldica que observamos en nuestros cuencos. Por lo tanto, las piezas Talaverana Heráldica - Cuenco I están relacionadas con fray Alonso de Medina y Salizanes. Según nuestra hipótesis, en los cuencos se ha segmentado el motivo heráldico. Esta división puede ser explicada por diferentes causas; quizá la principal fuese la dificultad del pintor cerámico para desarrollar una decoración tan compleja con cuatro cuarteles en una superficie tan pequeña como es el fondo de un cuenco de apenas unos centímetros. Ello haría que el pintor dividiese el escudo en partes, desarrollándolas de manera individual en los cuencos o piezas de menor tamaño. El motivo heráldico pudo estar pintado totalmente en piezas de mayores dimensiones como platos, fuentes o jarras no conservados.

De este modo, los cuencos asturianos se suman a los cuatro blasones que se conservan de fray Alonso de Medina y Salizanes, todos ellos en la Mezquita-Catedral de Córdoba. Tres de ellos en la capilla de la Concepción, uno en la escultura funeraria que representa al prelado en actitud de oración y arrodillado, otro a la derecha del arco de entrada a la antecapilla y el tercero, en el frontispicio de la capilla hacia el exterior. El último, descubierto en 2009, se ha recuperado en la cubierta de la nave central de la mezquita fundacional de Abd al-Rahman $\mathrm{I}^{60}$.

En conclusión, la inscripción de los cuencos y los dos motivos heráldicos que en ellos toman forma, pertenecen a fray Alonso de Medina y Salizanes. El obispo realizaría el encargo de estas piezas a algún alfar de Talavera de la Reina, coincidiendo con la fase en la que se desarrolla la serie heráldica Austria. La fecha de fabricación debe coincidir con el inicio de su etapa al frente del obispado de Oviedo (1675-1685). Puede que el aumento de las producciones provenientes de Talavera de la Reina que se observa en Asturias en el siglo XVII se deba a fray Alonso de Medina y Salizanes que trae consigo este encargo y probablemente otras piezas talaveranas ${ }^{61}$. La influencia que un obispo puede ejercer sobre sus feligreses en el seiscientos es grande y no 
parece descabellado que el menaje de mesa por él utilizado marcase una moda a imitar en Oviedo y el resto de la región.

\section{b. Una vajilla para una familia}

Entre las piezas procedentes de las excavaciones en la calle Schultz ${ }^{62}$ de Oviedo destaca, por ser la representación material de todo un linaje, la tipología Talaverana Heráldica - Plato I63 (Fig. 5. A). Estamos ante una pieza heráldica de ámbito familiar, en la que junto a la enseña del escudo aparece el nombre de la familia De la Madrid. Todo indica que estamos ante una producción de Talavera de la Reina fabricada en el siglo XVII, en torno a mediados de siglo, dentro de la serie heráldica Austria ${ }^{4}$. Nos encontramos, probablemente, ante el único ejemplar conservado de una vajilla que algún miembro de la familia De la Madrid encargó en Talavera de la Reina. Este hallazgo ejemplifica el poder económico adquirido por algunas familias nobles durante el siglo XVII en Asturias.

La casa y linaje De la Madrid es una familia importante desde el final de la Edad Media y durante toda la Edad Moderna. Tiene su asiento en la localidad de Lamadrid, en el Valle de La Valdáliga ${ }^{65}$, actual municipio de Valdáliga, en Cantabria. La rama De la Madrid más importante para Asturias será la que se afinque en Llanes, concretamente los Gómez De la Madrid. Diferentes miembros de esta familia serán procuradores y representantes de Llanes en la Junta del Principado. Nos referimos a Pedro De la Madrid, que viaja a Oviedo al menos en 1628 y 1631, y a Pedro Gómez De la Madrid, que en 1647 y 1652 está en Oviedo formando parte de la Junta 66 .
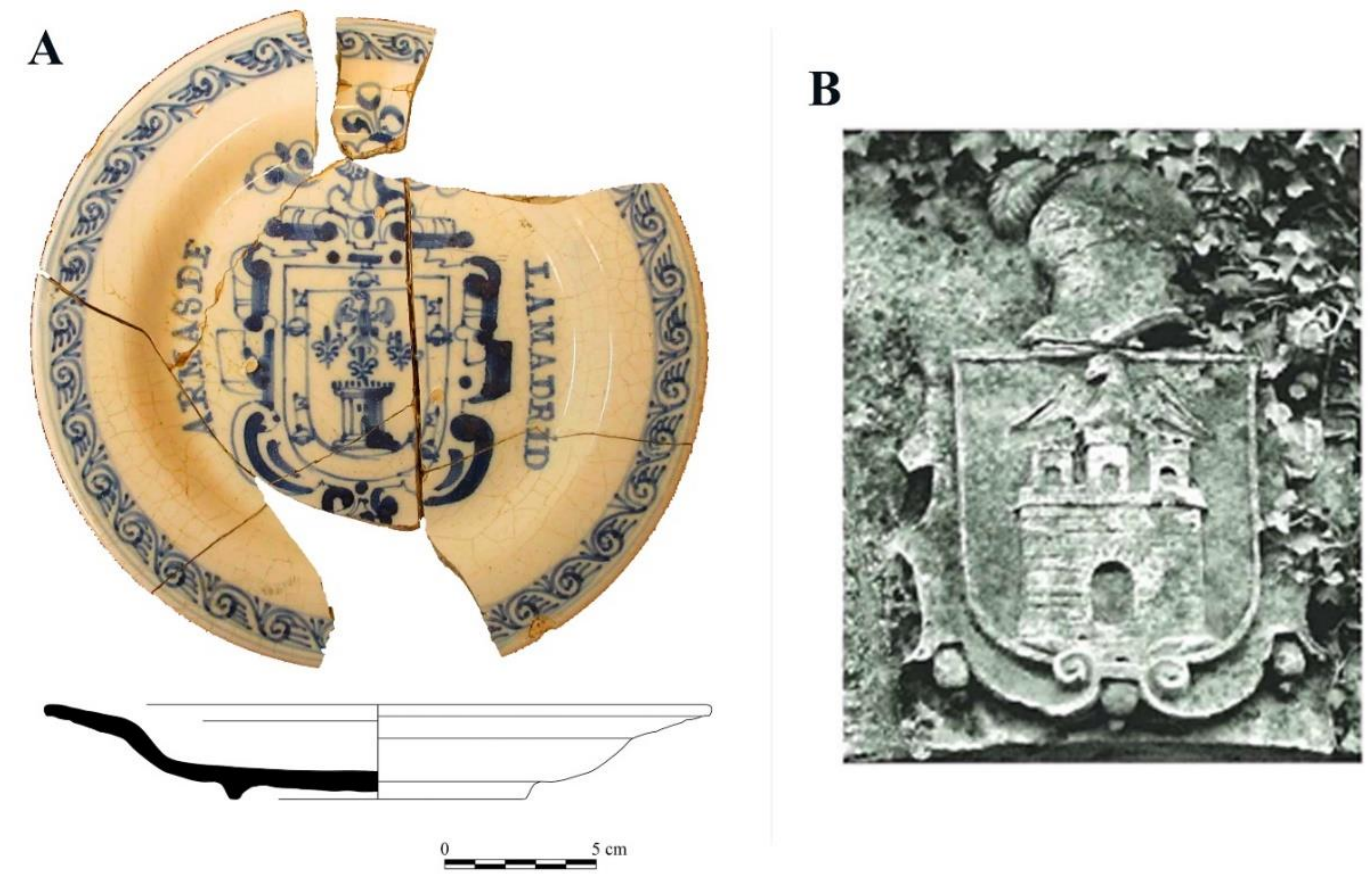

Fig. 5. A: Dibujo, acabado y decoración de Talaverana Heráldica - Plato I. Fig. 5. B: Escudo con armas de la Madrid, en Lamadrid, Cantabria (GONZÁLEZ ECHEGARAY, María del Carmen, Escudos de Cantabría..., cit., fig. 285). 
Analizando el escudo, las primitivas armas de la casa De la Madrid en el Valle de La Valdáliga, estarían compuestas por "un castillo mazonado y donjonado y sobre el homenaje águila explayada"67 (Fig. 5. B). Al pasar el escudo a otros valles "va aumentando el campo con otros aditamentos, como son tres torres, flores de lis, etc."68. En el siglo XVIII, el escudo de la casa de armas De la Madrid se hace más complejo. Por lo que respecta a la rama De la Madrid afincados en Llanes, que son concretamente Gómez De la Madrid, en 1773 su escudo de armas se "pinta por armas tres Castillos. O torres sobre agua, el de medio es mayor, y sobre cada uno una flor de lis y entre Castillo y Castillo una estrella, y una Águila a la puerta del Castillo de medio todo en campo rojo; Estas son las armas antiguas en cada uno de ellos"69. En 1783 se describe el escudo de armas compuesto por "tres castillos en campo rojo y en el del medio un águila coronada y en dicho campo sobre los castillos tres flores de lis y dos estrellas, orleado el escudo con su morrión que igualmente mandamos a dicho pintor nos le pintare y al escribano nos diere testimonio"70. Por su parte García Mijares, en 1893, al referirse a la casa y linaje De la Madrid, señala que su escudo "pinta por armas tres castillos ó torres sobre agua, el del medio es mayor, y sobre cada uno una flor de lis, y entre castillo y castillo una estrella, y un águila á la puerta del castillo del medio, todo en campo rojo. Estas son las armas antiguas que han usado pintar los dueños de la casa. Otros añaden cinco calderos con una bandera en cada uno de ellos"71. Desconocemos en qué datos se basa García Mijares para afirmar que se añaden al escudo cinco calderos con una bandera, elementos que observamos en nuestro plato. Este motivo se corresponde con el linaje de los Calderón, originarios de Cantabria. En Treceño, una localidad de la Valdáliga, se conserva un escudo con las armas De la Madrid y de Calderón, aunque la composición es diferente ${ }^{72}$.

El escudo representado en la tipología Talaverana Heráldica - Plato I no es exactamente el mismo que utiliza la rama de los Gómez De la Madrid afincados en Llanes y con relaciones con la Junta del Principado durante el siglo XVII. Los escudos De la Madrid conservados en Cantabria aparecen más sintetizados, presentando mayores similitudes con nuestro plato. Si a esto le sumamos que las cinco calderas con pendones están en relación con el linaje de los Calderón, originarios de Cantabria, muy probablemente el dueño de nuestro plato pertenecería a una rama De la Madrid relacionada con Valdáliga o San Vicente de la Barquera.

De todo lo expuesto se concluye que el encargo de Talaverana Heráldica - Plato I se realizó a Talavera de la Reina en el siglo XVII por parte de algún miembro de la familia De la Madrid de la vertiente cántabra. Probablemente relacionado con la ciudad de Oviedo y su cabildo. Por el momento, tampoco podemos desligarlo totalmente de la rama de los Gómez De la Madrid, de los que tenemos constancia de diferentes viajes a Oviedo en el siglo XVII. Lo que está claro es que este linaje tenía un gran poder en el seiscientos y utiliza parte de su capital para encargar una serie de piezas cerámicas a Talavera de la Reina y plasmar en ellas el escudo heráldico de su familia. Por lo tanto, no nos encontramos ante un simple plato, sino que estamos ante la plasmación material del poder de una familia, que utiliza la cerámica como un instrumento en el que reflejar su estatus dominante. 


\section{c. Un plato en apoyo de Guillermo III de Orange-Nassau}

Entre el material estudiado se encuentra una pieza cerámica con una gran carga política, puesto que se trata de un plato con la efigie del rey Guillermo III de Orange-Nassau. Se encontraba entre los materiales de la fosa séptica de la casa Carbajal Solís, en uso hasta finales del siglo XVII ${ }^{73}$. Se trata de la tipología Holandesa Faience - Plato VI ${ }^{74}$ elaborada en Holanda en la segunda mitad del siglo XVII ${ }^{75}$ (Fig. 6).
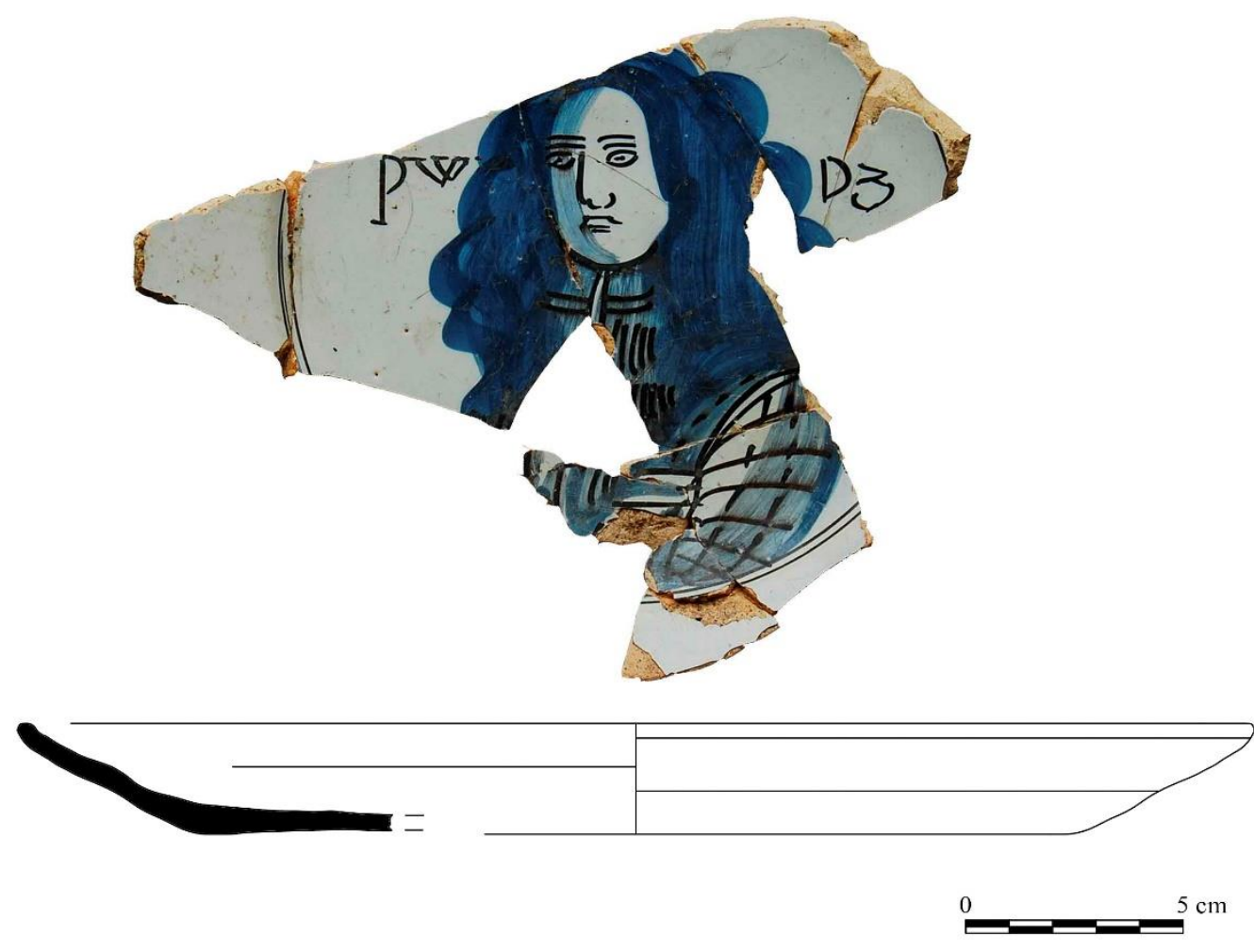

Fig. 6. Dibujo, acabado y decoración de Holandesa Faience - Plato VI.

Las letras que observamos de dos en dos a ambos lados de la figura son $P W$ D3. Estas letras son el acrónimo de Prins Willem Den 3de, que no es otro que Guillermo III de Orange-Nassau (1650-1702). Por lo tanto, la figura que aparece en este plato es la del líder del ejército de los Países Bajos que, en 1672 se convirtió en estatúder, un cargo político con poder ejecutivo sobre las provincias de Holanda, Zelanda y Utrecht. Tras la Revolución Gloriosa de 1689, Guillermo III de Orange-Nassau es coronado rey de Inglaterra e Irlanda, como Guillermo III, y rey de Escocia, como Guillermo II. Se trata, por tanto, de una figura muy importante en el panorama político europeo.

Después de su coronación, puede aparecer en piezas cerámicas junto a su esposa, María II de Inglaterra, con las iniciales $K W$ y $K M$, es decir, King William y King Mary76. Las cerámicas con las imágenes de Guillermo III de Orange-Nassau, tanto como príncipe o como rey, son un ejemplo de la propaganda que la casa de Orange realizaba en los Países Bajos y de la fuerza política de sus partidarios. En Holanda, la fabricación de platos con figuras políticas se detuvo entre finales del siglo XVII e inicios del siglo XVIII 77 . A finales del setecientos se retomaron de nuevo este tipo de producciones a 
causa de las tensiones políticas entre los partidarios de la familia Orange y la facción contraria o "patriotas"78. Además de en los Países Bajos, en Inglaterra estas piezas son relativamente comunes y nacen con un carácter conmemorativo, dado que en ellas se pintaban generalmente retratos reales. En las Islas Británicas se harán populares durante el reinado de Carlos I (1625-1649) y fueron producidas bajo los sucesivos monarcas hasta principios del siglo XVIII79. De hecho, hay autores que llegan a referirse a estas tipologías como "piezas políticas" 80 .

Como conclusión a todo lo expuesto, nos encontrarnos ante una producción de la segunda mitad del siglo XVII teñida de significado político. No es un simple plato en el que comer o con el que adornar la pared de una casa o un mueble, sino que este plato indica que su portador es partidario de la casa Orange y de Guillermo III de Orange-Nassau. Cabría preguntarse si los habitantes de la casa Carbajal Solís sabían quién aparecía dibujado en este plato que formaba parte de su menaje. Es lógico pensar que los poseedores de este plato lo ignoraban y lo apreciaban como un plato singular y lujoso.

\section{A modo de conclusión}

Del estudio llevado a cabo sobre 3.220 piezas cerámicas halladas en excavaciones arqueológicas en Asturias, se advierte cómo las élites van a demandar de manera creciente unos bienes de lujo para seguir los dictámenes de la moda europea. La vajilla de mesa se configura como una vajilla propagandística que refleja y transmite la condición social y económica de sus dueños. Estas vajillas estarán configuradas por piezas francesas, sevillanas, italianas, holandesas, talaveranas y portuguesas, entre otras muchas. Junto a estas élites el ascenso de una clase comercial y artesanal urbana también fue clave en el aumento del consumo de cerámica importada que hemos atestiguado en los siglos XV, XVI y, sobre todo, XVII.

Observamos además la proliferación de unas piezas cerámicas de carácter singular como las analizadas en la última parte de este trabajo. Tal y como señalamos, en estos casos estamos ante la plasmación material del poder de unas élites tanto religiosas como civiles, que van a utilizar la cerámica como un instrumento en el que reflejar su estatus dominante. Además de consumir piezas cerámicas importadas, las élites pueden acceder a producciones exclusivas e incluso al encargo directo de vajillas. De este modo ha quedado atestiguado que en Asturias durante la Edad Moderna algunas producciones cerámicas son algo más que barro cocido. Son piezas que se han convertido en un símbolo de poder, prestigio y un elemento de diferenciación social.

\section{NOTAS}

\footnotetext{
${ }^{1}$ Esta investigación toma como base el Capítulo V.3. Significación social de las cerámicas de BUSTO ZAPICO, Miguel, Cerámica de importación en el Principado de Asturias entre la Baja Edad Media y la primera Edad Moderna, Oviedo, Universidad de Oviedo, Tesis Doctoral inédita, 2018. Esta Tesis Doctoral fue defendida en noviembre de 2018 obteniendo la calificación de Sobresaliente cum laude; los directores
} 
fueron la Dra. Yayoi Kawamura (Universidad de Oviedo) y el Dr. Enrico Cirelli (Università di Bologna). El tribunal estuvo formado por el Catedrático José Avelino Gutiérrez González (Universidad de Oviedo), el Catedrático Alfonso Pleguezuelo Hernández (Universidad de Sevilla), el Dr. Enrico Cirelli (Università di Bologna) y el Dr. Antonio Vannugli Nicolé (Università degli Studi del Piemonte Orientale). Agradecemos a todos ellos la ayuda prestada y sus aportaciones tanto en el proceso de investigación como en el acto de defensa. Queremos además señalar la ayuda recibida del Dr. Guillermo Fernández Ortiz para el desarrollo del apartado 4.1. La vajilla del obispo y de Inés García-Navarro Aguirre para el 4.2. Una vajilla para una familia. La ayuda de Carmen de Hoyos González fue fundamental en el trabajo con los Padrones de Vecindad. La investigación ha sido subvencionada por el Gobierno del Principado de Asturias a través del Programa "Severo Ochoa" de Ayudas Predoctorales para la investigación y docencia en el Principado de Asturias.

${ }^{2}$ La muestra analizada es una selección del material cerámico analizado en: BUSTO ZAPICO, Miguel, Cerámica de importación en el..., cit.

${ }^{3}$ BUSTO ZAPICO, Miguel, Cerámica de importación en el..., cit., pp. 1361-1421; BUSTO ZAPICO, Miguel y GUTIÉRREZ GONZÁLEZ, José Avelino, "Cerámicas de importación en Oviedo (Asturias) entre los siglos XVI y XVII. Materiales procedentes de la casa Carbajal Solís", en Sautuola. Revista del Instituo de Prehistoria y Arqueología Sautuola, XXI, 2016, pp. 197-219; BUSTO ZAPICO, Miguel, "Los influjos estilísticos europeos en las producciones de cerámica asturiana de la Edad Moderna", en Liño. Revista Anual de Historia del Arte, 25, 2019, pp. 9-22.

${ }^{4}$ CARTA, Raffaella, Difusión e influencia de la producción de la cerámica Italiana entre la Baja Edad Media y la primera Edad Moderna. El caso de Granada, Granada, Universidad de Granada, Tesis Doctoral inédita, 2008, p. 81.

${ }^{5}$ VILLANUEVA ZUBIZARRETA, Olatz, "La ollería y alcallería en la cuenca del Duero a lo largo de la Edad Media y Moderna", en Manual de Cerámica Medieval y Moderna, Madrid, Sección de Arqueología del CDL de Madrid, 2011, pp. 87-116.

${ }^{6}$ BUSTO ZAPICO, Miguel, "Cerámica de producción local en Asturias entre los siglos XVI y XVII, procedente de las excavaciones de la casa Carbajal Solís (Oviedo, Asturias)", en Boletín de Letras del Real Instituto de Estudios Asturianos, 69, 185-186, 2015, pp. 33-64.

${ }^{7}$ BUSTO ZAPICO, Miguel, Cerámica de importación en el..., cit., pp. 1366-1372.

${ }^{8}$ CARTA, Raffaella, Difusión e influencia ..., cit., pp. 76-83.

${ }^{9}$ GOLDTHWAITE, Richard A., "Il mondo economico e sociale della maiolica italiana nel Rinascimento", en Faenza, LXXXIII, IV-VI, 1997, pp. 176-202.

${ }^{10}$ GOLDTHWAITE, Richard A., "Il mondo economico...", cit., pp. 190-194.

11 GOLDTHWAITE, Richard A., "Il mondo economico...", cit., pp. 190-194; VILLANUEVA ZUBIZARRETA, Olatz, "La ollería y alcallería...", cit., p. 97.

12 MORATINOS GARCÍA, Manuel y VILLANUEVA ZUBIZARRETA, Olatz, "Usos, modas y cambios: el gusto por los "barros de Portugal" en la cuenca del Duero y sus réplicas hispanas durante el Antiguo Régimen", en Boletín del Seminario de Estudios de Arte y Arqueología, LXXIX, 2013, pp. 153-175.

${ }^{13}$ MORATINOS, Manuel y VILLANUEVA, Olatz, "Usos, modas...”, cit., p. 154.

${ }^{14}$ CARTA, Raffaella, Difusión e influencia ..., cit., p. 82.

${ }^{15}$ GARCÍA FUEYO, Beatriz, Alonso Antonio de San Martín, obispo de Oviedo y Cuenca e hijo de Felipe IV, Oviedo, Universidad de Oviedo, 2012, p. 80, nota 431.

${ }^{16}$ CARTA, Raffaella, Difusión e influencia ..., cit., p. 113.

${ }^{17}$ BUSTO ZAPICO, Miguel, GUTIÉRREZ GONZÁLEZ, José Avelino y ESTRADA GARCÍA, Rogelio, "Las lozas de la casa Carbajal-Solís. Punto de encuentro entre el Mediterráneo y el Norte de Europa", en Actas do X Congresso Internacional a Cerâmica Medieval no Mediterrâneo, Silves, Câmara Municipal De Silves \& Campo Arqueológico de Mértola, 2015, pp. 466-472.

${ }^{18}$ BUSTO ZAPICO, Miguel, Cerámica de importación en el..., cit., pp. 1065-1079.

${ }^{19}$ BUSTO ZAPICO, Miguel, Cerámica de importación en el..., cit., pp. 1081-1085.

${ }^{20}$ MORATINOS, Manuel y VILLANUEVA, Olatz, "Usos, modas...”, cit., p. 154.

${ }^{21}$ BUSTO ZAPICO, Miguel, Cerámica de importación en el..., cit., pp. 1380-1408.

${ }^{22}$ CARTA, Raffaella, Difusión e influencia..., cit., p. 82.

${ }^{23}$ NEWSTEAD, Sarah y CASIMIRO, Tânia Manuel, "A Cerâmica Portuguesa no Atlântico Norte (Séculos XVII-XVIII) o iniciar de um projecto de investigação", en Almadan online, 19, 2015, pp. 64-69. 
${ }^{24}$ BUSTO ZAPICO, Miguel, Cerámica de importación en el..., cit., pp. 558-598.

${ }^{25}$ NEWSTEAD, Sarah y CASIMIRO, Tânia Manuel, "A Cerâmica Portuguesa...", cit., p. 68.

${ }^{26}$ PLEGUEZUELO HERNÁNDEZ, Alfonso, "Cerámicas para agua en el barroco español: Una primera aproximación desde la literatura y la pintura", en Ars Longa, 2000, pp. 123-138.

${ }^{27}$ MORATINOS, Manuel y VILLANUEVA, Olatz, "Usos, modas...”, cit., p. 163.

${ }^{28}$ PLEGUEZUELO HERNÁNDEZ, Alfonso, "Cerámicas para agua...", cit., p. 130.

${ }^{29}$ MORATINOS, Manuel y VILLANUEVA, Olatz, "Usos, modas...”, cit., pp. 163-164.

${ }^{30}$ A.M.O.: Padrón de vecindad de la ciudad de Oviedo de 1664. Signatura B-042-1, f. 27r.

${ }^{31}$ A.M.O.: Padrón de vecindad de la ciudad de Oviedo de 1664. Signatura B-042-1, f. 30r.

${ }^{32}$ A.M.O.: Padrón de vecindad de la ciudad de Oviedo de 1664. Signatura B-042-1, f. 29a.

${ }^{33}$ A.M.O.: Padrón de vecindad de la ciudad de Oviedo de 1664. Signatura B-042-1, f. 30r-31a.

${ }^{34}$ A.M.O.: Padrón de vecindad de la ciudad de Oviedo de 1670. Signatura B-042-2, imagen 26.

35 A.M.O.: Padrón de vecindad de la ciudad de Oviedo de 1670. Signatura B-042-2, imagen 26. A.M.O.: Padrón de vecindad de la ciudad de Oviedo de 1681. Signatura B-043-1-1, imagen 14.

${ }^{36}$ A.M.O.: Padrón de vecindad de la ciudad de Oviedo de 1692. Signatura B-043-3-2, f. 10r.

${ }^{37}$ BUSTO ZAPICO, Miguel, Cerámica de importación en el ..., cit., pp. 224-227, 319-320, 458-460, 739740, 845-848, 1339-1340.

${ }^{38}$ A.M.O.: Padrón de vecindad de la ciudad de Oviedo de 1664. Signatura B-042-1, f. 28r. A.M.O.: Padrón de vecindad de la ciudad de Oviedo de 1670. Signatura B-042-2, imagen 23.

${ }^{39}$ A.M.O.: Padrón de vecindad de la ciudad de Oviedo de 1664. Signatura B-042-1, f. 29r.

${ }^{40}$ A.M.O.: Padrón de vecindad de la ciudad de Oviedo de 1664. Signatura B-042-1, f. 30a.

${ }^{41}$ A.M.O.: Padrón de vecindad de la ciudad de Oviedo de 1664. Signatura B-042-1, f. 30r.

${ }^{42}$ A.M.O.: Padrón de vecindad de la ciudad de Oviedo de 1670. Signatura B-042-2, imagen 23.

${ }^{43}$ A.M.O.: Padrón de vecindad de la ciudad de Oviedo de 1674. Signatura B-042-3, imagen 39.

${ }^{44}$ A.M.O.: Padrón de vecindad de la ciudad de Oviedo de 1681. Signatura B-043-1-1, imagen 14.

${ }^{45}$ A.M.O.: Padrón de vecindad de la ciudad de Oviedo de 1686. Signatura B-043-2-2, f. 10a.

${ }^{46}$ A.M.O.: Padrón de vecindad de la ciudad de Oviedo de 1664. Signatura B-042-1, f. 31a.

${ }^{47}$ BUSTO ZAPICO, Miguel, Cerámica de importación en el..., cit., pp. 643-649.

48 A.M.O.: Padrón de vecindad de la ciudad de Oviedo de 1670. Signatura B-042-2, imagen 26.

${ }^{49}$ A.M.O.: Padrón de vecindad de la ciudad de Oviedo de 1692. Signatura B-043-3-2, f. 9r.

${ }^{50}$ A.M.O.: Padrón de vecindad de la ciudad de Oviedo de 1692. Signatura B-043-3-2, f. 9r.

${ }^{51}$ FERNÁNDEZ BUELTA y José, HEVIA GRANDA, Víctor, Ruinas del Oviedo Primitivo. Historia y secuencias de unas excavaciones, Oviedo, Instituto de Estudios Asturianos, 1984.

52 BUSTO ZAPICO, Miguel, Cerámica de importación en el..., cit., pp. 449-454.

53 FERNÁNDEZ BUELTA, José y HEVIA GRANDA, Víctor, Ruinas del Oviedo..., cit.; BUSTO ZAPICO, Miguel, Cerámica de importación en el..., cit., pp. 105-107.

${ }^{54}$ GONZÁLEZ ZAMORA, César, Talaveras, Madrid, Grupo Antiquitas S.L., 2004, p. 151.

55 TUÑON, Juan José, "Los obispos ovetenses del siglo XVII y las celebraciones sinodales", en Historia de las diócesis españolas, 17: Iglesias de Oviedo y León, Madrid, BAC, 2016, pp. 245-258.

${ }^{56}$ GÓMEZ BRAVO, Juan, Catálogo de los Obispos de Córdoba y breve noticia histórica de su Iglesia Catedral y Obispado, Vol. Tomo II, Córdoba, 1778, pp. 576-606.

57 JORDANO BARBUDO, María Ángeles, "La intervención de los obispos Mardones y Salizanes en la nave central de Abd al-Rahman I en la Mezquita-Catedral de Córdoba", en Ámbitos. Revista de estudios de Ciencias Sociales y Humanidades, 24, 2010, pp. 13-21; RAMÍREZ DE ARELLANO, Rafael, Inventario Monumental y Artístico de la Provincia de Córdoba, Córdoba, Diputación Provincial de Córdoba, 1983, p. 131; NIETO CUMPLIDO, Manuel, La Catedral de Córdoba, Cajasur, Córdoba, 1998, pp. 351-354.

58 GÓMEZ BRAVO, Juan, Catálogo de los Obispos..., cit., p. 707.

${ }^{59}$ MOLINERO MERCHÁN, Juan Andrés, La Mezquita-Catedral de Córdoba: Símbolos de poder. Estudio Histórico-Artístico a través de sus Armerías, Córdoba, Ayuntamiento de Córdoba: Universidad de Córdoba Servicio de Publicaciones, 2005, p. 483.

60 JORDANO BARBUDO, María Ángeles, "La intervención de los obispos ...", cit... 
${ }^{61}$ BUSTO ZAPICO, Miguel, Cerámica de importación en el..., cit., pp. 1393-1408.

${ }^{62}$ GARCÍA DE CASTRO VALDÉS, César, "Informe sobre los sondeos arqueológicos realizados en el n³ de la calle Shultz, de Oviedo (antiguo Colegio San Isidoro)", en Memoria Arqueológica Inédita, 2000; GARCÍA DE CASTRO VALDÉS, César, "Informe sobre las excavaciones arqueológicas realizadas en el $\mathrm{n}^{\circ} 3$ de la calle Shultz (antiguo Colegio San Isidoro) y en la misma calle Shultz de la ciudad de Oviedo (febrero 2000 - Abril 2001)", en Memoria Arqueológica Inédita, 2001.

${ }^{63}$ BUSTO ZAPICO, Miguel, Cerámica de importación en el..., cit., pp. 455-458.

${ }^{64}$ GONZÁLEZ ZAMORA, César, Talaveras, cit., p. 151.

${ }^{65}$ GARCÍA MIJARES, Manuel, Apuntes históricos, genealógicos y biográficos de Llanes y sus hombres 1893, Llanes, El Oriente de Asturias, 1990, pp. 176-186.

${ }^{66}$ CANELLA Y SECADES, Fermín, Historia de Llanes y su concejo, Llanes, 1896.

${ }^{67}$ GONZÁLEZ ECHEGARAY, María del Carmen, Escudos de Cantabría. Tomo IV. Asturias de Santillana y el Bajo Asón, Vitoria, Institución cultural de Cantabria, 1981, p. 159.

${ }^{68}$ GONZÁlEZ ECHEGARAY, María del Carmen, Escudos de Cantabría ..., cit., p. 159.

${ }^{69}$ AHA, PH, Caja 11471 /19. Casas de Rivero y Gómez la Madrid. Año 1773. Los documentos los aporta Joaquín de Rivero Gómez De la Madrid. Agradecemos a Inés García-Navarro Aguirre que haya compartido con nosotros esta información y la transcripción del documento.

${ }^{70}$ AHN, OM, Caballeros de Alcántara, exp. 2216 (microfilme, pos. 723) Fol. 42 v. Al igual que en el caso anterior debemos esta información a Inés García-Navarro Aguirre.

${ }^{71}$ GARCÍA MIJARES, Manuel, Apuntes históricos..., cit., p. 176.

${ }^{72}$ GONZÁLEZ ECHEGARAY, María del Carmen, Escudos de Cantabría ..., cit., p. 170, Fig. 314.

${ }^{73}$ ESTRADA GARCÍA, Rogelio, "Avance sobre los sondeos arqueológicos preliminares del Proyecto de Ampliación del Museo de Bellas Artes de Asturias", en Memoria Arqueológica Inédita, 2003; BUSTO ZAPICO, Miguel, GUTIÉRREZ GONZÁLEZ, José Avelino y ESTRADA GARCÍA, Rogelio, "Las lozas de la casa...", cit., pp. 466-472; BUSTO ZAPICO, Miguel y GUTIÉRREZ GONZÁLEZ, José Avelino, "Cerámicas de importación en Oviedo...", cit., pp. 197-219.

${ }^{74}$ BUSTO ZAPICO, Miguel, Cerámica de importación en el..., cit., pp. 928-930.

75 GAWRONSKI, Jerzy, JAYASENA, Ranjith, LAGERWEIJ, Ab, OSTKAMP, Sebastiaan, TOUSAIN, Ron y VEERKAMP, Jorgen, "Catalogue of archaeological ceramics from Amsterdam 1175-2011", en Amsterdam Ceramics. A city's history and an archaeological ceramics catalogue 1175-2011, Spruijt, Uitgeverij Bas Lubberhuizen, 2012, pp. 242, 323.

${ }^{76}$ SNODIN, Michael, Horace Walpole`s Strawberry Hill, Londres, 2009, p. 331.

77 STELLINGWERF, Wytze, "De patriot achter de pot. Keramiek en politiek in de patriottentijd", en Arheologie in Nederland, 4, 2017, pp. 34-43.

${ }^{78}$ STELLINGWERF, Wytze, "De patriot achter de pot...", cit., pp. 34-43.

79 GRIGSBY, Leslie Brown, The Longridge collection of English slipware and delftware, Londres, Jonalhan Horne Publications, 2000, pp. 10-19.

${ }^{80}$ FAY-HALLÉ, Antoinette y LAHAUSSOIS, Christine, La Faïence Européenne au XVIIème siècle. Le triomphe de Delft, Réunion des Musées Nationaux, París, 2003, pp. 240-241.

Fecha de recepción: 27 de marzo de 2019

Fecha de revisión: 16 de mayo de 2019

Fecha de aceptación: 4 de julio de 2019 